\title{
Review of Different Queuing Disciplines in VOIP, Video Conferencing and File Transfer
}

\author{
Rajeev Sharma ${ }^{1}$, Sukhjit Singh Sehra ${ }^{2}$, Sumeet Kaur Sehra ${ }^{3}$ \\ M.Tech. (CSE) Student, Guru Nanak Dev Engineering College, Ludhiana, Punjab, India ${ }^{1}$ \\ Assistant Professor, Guru Nanak Dev Engineering College, Ludhiana, Punjab, India ${ }^{2,3}$
}

\begin{abstract}
Now a day Internet only put up best effort service. Traffic is transmitting as earliest as possible, but during transmission, there is no guarantee of timelines or real delivery of packets. With the swiftly transformation of the Internet into a commercial infrastructure, demands for a quality of service have developed in rapid rate. People of the present world are very much depending upon the various network services like VOIP, Video conferencing and File Transfer [6,7]. Various categories of Traffic Management systems are used in those services. Queuing is one of the very important mechanisms in traffic management system. Each router in the network must implement some queuing discipline that control how packets are buffered while waiting to be transmitted. The main aim of this paper is to highlight quality of service (QoS) analysis using different queuing disciplines.
\end{abstract}

Keywords: FIFO queue, Priority queue, Weighted-fair queue, QoS, MWRR, DWRR, MDRR

\section{INTRODUCTION}

Queuing is the mathematical study of waiting lines, or queues. In queuing theory a model is constructed so that queue lengths and waiting times can be predicted. Queuing theory is generally considered a branch of operations research because the results are often used when making business decisions about the resources needed to provide a service. The current Internet is based on IP protocol and supports only best effort services. With the rapid growth of Internet during the last years, IP networks are expected to support not only typical services like ftp and email, but also real-time services and video application.

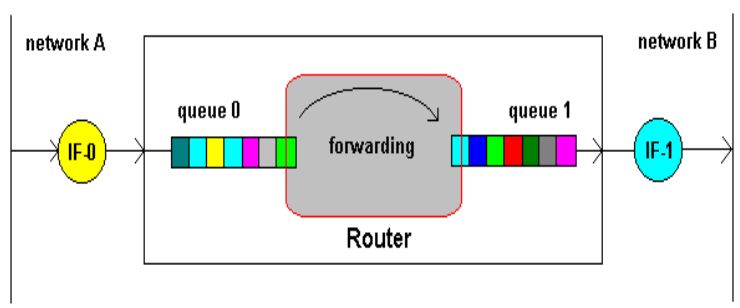

Figure 1: Process of queuing

The traffic characteristics of these applications require a certain Quality of Service (QoS) from the network in terms of bandwidth and delay requirements [1]. The biggest problems in a network are related to the allocation of network resources, as buffers and link band width, to different users. A limited amount of resources has to be shared among many different competing traffic flows in an efficient way in order to maximize the performance and the use of the order to transmit those packets, and the packets in other queue is again empty. When a packet is sent out an interface, the priority queues on that interface are scanned for packets in descending order for priority. Firstly the high priority queue is scanned, then the medium priority queue and then so on. The packet at the head of the highest queue is chosen for transmission. This process is repeated every time when a packet is to be sent. In order to complete different quality of service, the network should manage a better quality of service to provide satisfactory output to the user [2]. If the traffic of the network is due to congestion, it reduces the network performance. So the different queuing disciplines are applied on the network to different application to improve the performance of the network.

\section{QUEUING DISCIPLINES}

As part of the resource allocation process, each router must go through by some queuing discipline that governs how packets are buffered while waiting to be transmitted. Various queuing disciplines can be used to control which packets get transmitted (bandwidth allocation) and which packets get dropped (buffer space).The queuing discipline also affects the latency experienced by a packet, by determining how long a packet waits to be transmitted. Examples of the common queuing disciplines are first-infirst-out (FIFO) queuing, priority queuing (PQ), and weighted-fair queuing (WFQ)[3]. Various queuing disciplines can be used to control which packets get transmitted and which packets which packets get dropped.

The queuing disciplines are

1. First-in-first-out (FIFO) queuing.

2. Priority Queuing $(\mathrm{PQ})$

3. Weighted-Fair queuing. (WFQ)

4. Custom queuing

5 MWRR

6 DWRR

7 MDRR

A. First-in-first-out (FIFO) queuing

FIFO is an short form for First In First Out .This appearance describes the principle of a queue or first-come first serve behavior: what comes in first is handled first, what comes in next waits until the first is finished etc. 


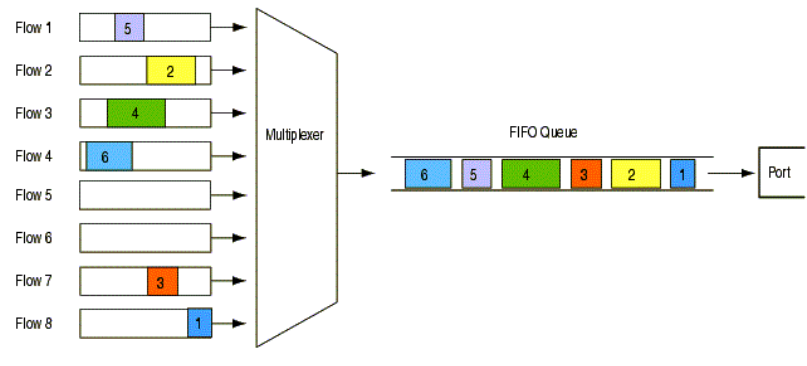

Figure 2: File transfer on interactive traffic with FIFO

Thus it is similar to the behavior of persons "standing in a line" or "Queue" where the persons leave the queue in the order they arrive. First In First out (FIFO) is the most essential queuing discipline. In FIFO Queuing all packets are treated equally by placing them into a single queue, then servicing them in the same order they were placed in the queue. FIFO queuing is also known to as First Come First Serve (FCFS) queuing.

\section{B. Priority Queuing $(P Q)$}

In Priority queue, there are lots of queue that is given to the network interface and each queue has a priority level. A queue with top most priority is processed first than a queue

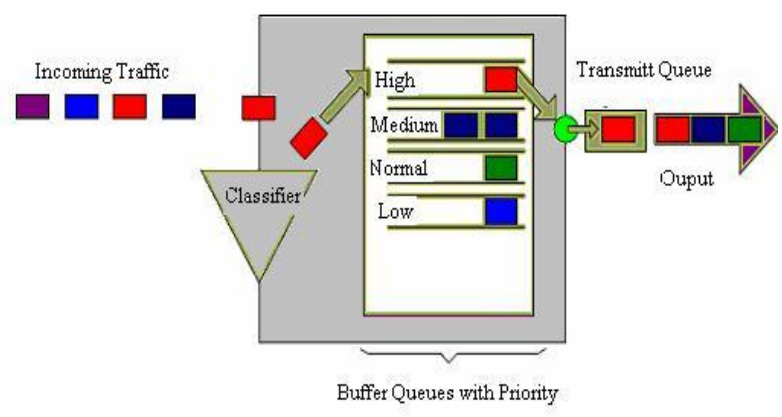

figure 3: Priority Queuing

with lower priority. Priority Queuing has four reconfigured queues, high medium, normal and low priority queue. By defaulting each of these queues has 20, 40, 60 and 80 packets capacity [1]. If packets reach your destination in the high queue then priority queuing drops everything its doing in order to transmit those packets. When a packet is sent out an interface, the priority queues on that interface are scanned for packets in descending order for priority. The high priorities Queue is scanned first, then the medium priority queue and then so on. The packet at the head of the highest queue is chosen for transmission. This process is repeated every time when a packet is to be sent.

\section{Weighted-Fair queuing. (WFQ)}

WFQ is the best known and the most studied queuing discipline. It assigns a queue for each flow and applies priority (or weights) to identified traffic to determine how much bandwidth each flow is allowed relative to others the maximum length of a queue is defined by the length limit
[4]. When a queue is longer the limit packets are dropped In QoS, a flow-based queuing algorithm that schedules low-volume traffic first, while letting high-volume traffic share the remaining bandwidth.

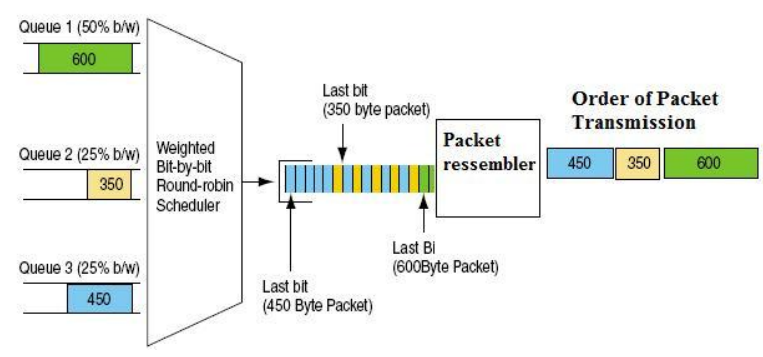

Figure 4: Weighted Fair Queuing

This procedure is done by provide a weight to every flow, In this type of queue, where lower weight are the highest priority

\section{Custom Queuing}

In Custom Queuing (CQ) is a Congestion Management technique which is round-robin like where frames from each queue are serviced

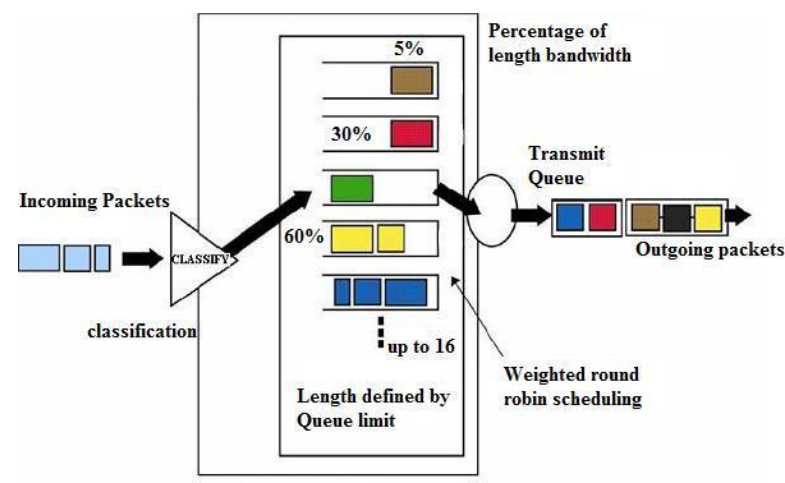

Figure 5: Custom Queuing

until a byte-counter limit threshold is met. Once this threshold is met, the frames from next queue are serviced.

\section{E. $\quad$ Modified Weighted Round Robin (MWRR)}

In MWRR queuing discipline allows high priority packets to cut front of the line. MWRR

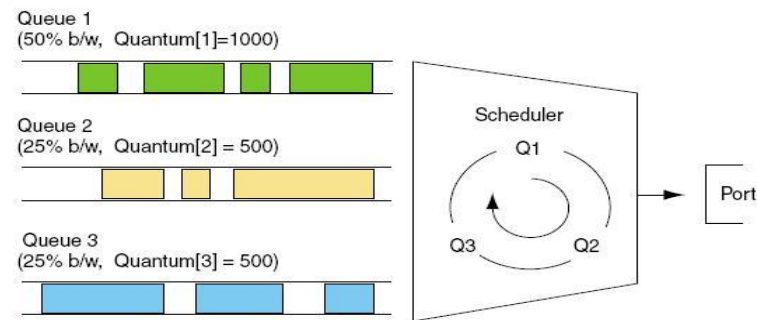

Figure 6: Modified Weighted Round Robin is used in Cisco switches. A weight is assigned to each queue in MWRR. This technique uses variable-sized packets to be serviced [8]. 


\section{F. $\quad$ Deficit Weighted Round Robin (DWRR)}

In DWRR, A weighted round-robin (WRR) method that uses a deficit counters. A maximum packet size number is subtracted from the packet length,

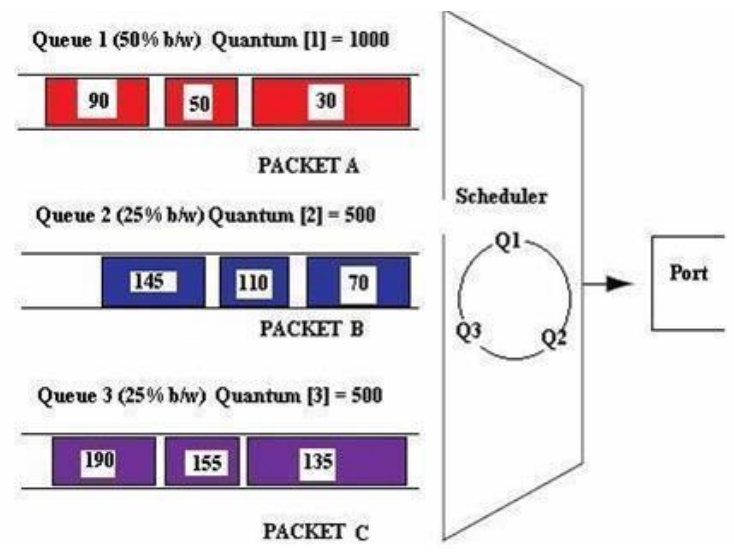

Figure 7: Deficit Weighted Round Robin

and packets that exceed that number are held back until the next visit of the scheduler.

\section{G. $\quad$ MDRR (Modified Deficit Round Robin)}

MDRR (Modified Deficit Round Robin), With MDRR configured as the queuing strategy, non-empty queues in a round-robin fashion. Each time a queue is served, a fixed amount of data is dequeued. The algorithm then services the next queue. When a queue is served, MDRR keeps track of the number of bytes of data that was dequeued
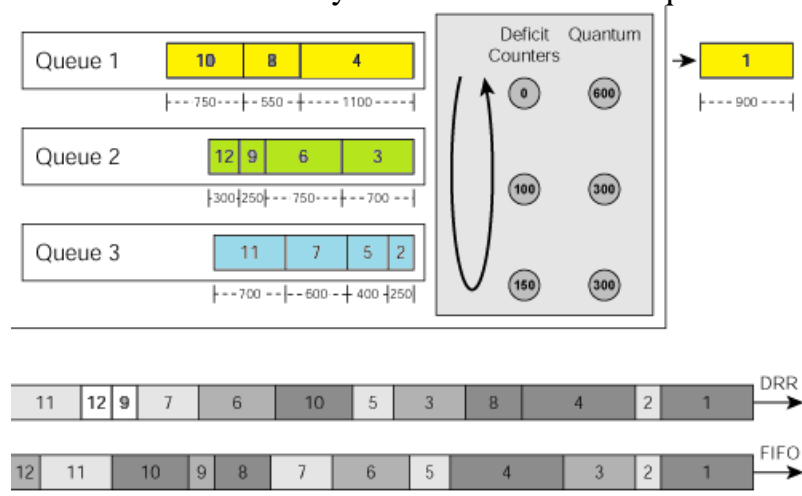

Figure 8: Modified Deficit Round Robin

in excess of the configured value. In the next pass, when the queue is served again, less data will be dequeued to compensate for the excess data that was served previously served one after anotherAs a result, the average amount of data dequeued per queue will be close to the configured value. In addition, MDRR maintains a priority queue that gets served on a preferential basis. MDRR is explained in greater detail in this section.

Each queue within MDRR is defined by two variables:

- $\quad$ Quantum value - This is the average number of bytes served in each round.

- Deficit counter - This is used to track how many bytes a queue has transmitted in each round. It is initialized to the quantum value.
Packets in a queue are served as long as the deficit counter is greater than zero. Each packet served decreases the deficit counter by a value equal to its length in bytes. A queue can no longer be served after the deficit counter becomes zero or negative. In each new round, the deficit counter of each non-empty queue is increased by its quantum value.

Here different queuing discipline in the routers can affect the performance of the applications and the utilization of the network resources

\section{APPLICATIONS OF QUEUING DISCIPLINES}

1 .Scaling up the arrival rate and service rate If we increase the arrival and service rates by the same factor then average number of customers in the system stays the same, while the average delay goes down

\section{Multiplexing several connections on one link}

3. Queues are a ubiquitous part of everyday life; common examples are supermarket checkout stations, help desk call centers, manufacturing assembly lines, wireless communication networks, and multi-tasking computers.

4. Queuing theory provides a rich and useful set of mathematical models for the analysis and design of service process for which there is contention for shared resources.

\section{CONCLUSIONS}

This paper review the different queuing discipline As the bulk of traffic and number of simultaneously active flows on Internet backbones increases, the problem of recognizing and addressing congestion within the network so the performance of the network is reduce There are two major ways in which routers can help in congestion control: by signaling congestion using either packet drops, and by cleverly managing its buffers. Today, Queuing is the modern in which routers can assist in congestion control. A particular form of queuing called fair queuing (FQ) achieves (weighted) fair allocation of bandwidth between flows. While FQ achieves weighted-fair bandwidth allocations, it requires per-flow queuing and per-flow state in each router in the network. The use of a queuing network model helps in extending the traffic model to incorporate the traffic. Thus, with help of actual road data, various combinations of routing probabilities between nodes can be used to model different vehicle types. This paper only gives incorporating more wellknown queuing network models. We identified different inputs and outputs for the present system and review the performance and capability of some important queuing techniques.

\section{REFERENCES}

1]. Mohammad, M., Golam, R., Mamun, K., "A Comparative Study of different queuing techniques in VOIP, Video conferencing and File transfer", Daffodil International University Journal Of Science And Technology, Volume 5, Issue 1, January 2010, pp: 37-47

[2] Sandeep, Rambir, J., Mandeep Singh, C., "Simulation \& Comparison of CSFQ, RED \& FRED Queuing Technique", International Journal of Soft Computing and Engineering (IJSCE) ISSN: 2231-2307, Volume-2, Issue-3, July 2012, pp: 392-396 


\section{International Journal of Advanced Research in Computer and Communication Engineering} Vol. 4, Issue 3, March 2015

[3] Payal, Richa, G., "Review on Optimization of Computer Networks Using QOS", International Journal of Advanced Research in Computer Science and Software Engineering, ISSN: 2277 128X, Volume 4, Issue 4, April 2014. pp:1003-1006

[4] Balasundaram, K., Velmurugan, T., Suresh R.,"Performance Analysis of Queuing Disciplines for Difference Services Using OPNET Tool", International Journal of Scientific Engineering and Technology, ISSN: 2277-1581Volume 3, Issue 1, 1 Jan 2014, pp : 47-50

[5]. Muhammad Aamir, Mustafa Zaidi and Husnain Mansoor, "Performance Analysis of DiffServ based Quality of Service in a Multimedia Wired Network and VPN effect using OPNET", International Journal of Computer Science Issues (IJCSI); May 2012.

[6]. Mitko Gospodinov, "The affects of different queuing disciplines over FTP, Video and VoIP Performance", in International Conference On Computer Systems and Technologies CompSysTech, 2004, pp. 19-1 - 19-5.

[7]. T. Velmurugan, Himanshu Chandra, and S. Balaji, "Comparison of Queuing Disciplines for Differentiated Services using OPNET", in International Conference On Advances in Recent Technologies in Communication and Computing (ARTCom'09), IEEE, October 2009, pp. 744- 746.

[8]. Sarhan M. Musa, Mahamadou Tembely, Matthew N. O. Sadiku,And Pamela Obliomon, "A Comparative Study of Different Queuing Scheduling Disciplines", in int. Journal of Engineering Research and Applications, ISSN : 2248-9622, Vol. 3, Issue 6, Nov-Dec 2013, pp.1587-1591 